COMMENT

DOI: 10.1057/s41599-018-0155-z

\title{
Considering transgender and gender nonconforming people in health communication campaigns
}

\author{
Ryan Combs (1) 1 , Monica Wendel ${ }^{1} \&$ T. Gonzales ${ }^{2}$
}

\begin{abstract}
Transgender and gender nonconforming (TGNC) people have become more visible and risen in the American public's consciousness in recent years. Despite this visibility and some political gains such as piecemeal advances in legal gender recognition and nondiscrimination protection, TGNC people continue to experience extensive health and social disparities. Notably, these disparities include a higher human immunodeficiency virus (HIV) burden, poorer mental health outcomes, and barriers to health care. Public health professionals in the United States have called for TGNC health inequities to be addressed and health communication is an important component of this effort. This article begins with an overview of the research evidence about the extent of TGNC disparities and contextualizes them sociopolitically. Then, we review a sample of existing health communication campaigns relating to breast, cervical, and testicular cancers through a TGNC lens. Finally, we make a case for considering TGNC people in the development and delivery of health communication campaigns. Recommendations are made for how to do this effectively. Suggested actions include reviewing existing materials for gender inclusivity, engaging communities to determine accurate and relevant messaging, collecting population-level demographic data on gender identity, and training employees using a health equity framework.
\end{abstract}

\footnotetext{
${ }^{1}$ Health Promotion \& Behavioral Sciences, University of Louisville School of Public Health \& Information Sciences, Louisville, KY 40202, USA. ${ }^{2}$ Center for Health Equity, Louisville Metro Department of Public Health \& Wellness, Louisville, KY 40202, USA. Correspondence and requests for materials should be addressed to R.C. (email: ryan.combs@louisville.edu)
} 


\section{Introduction}

ransgender and gender nonconforming (TGNC) people ${ }^{1}$, those whose gender identity does not correspond with the sex they were assigned at birth, have become more visible and risen in the public consciousness in recent years. More accurate data about the United States (U.S.) transgender population was collected through the 2014 Behavioral Risk Factor Surveillance System (BRFSS), leading researchers to double previous estimates of the population from 0.3 to $0.6 \%$, approximately 1.4 million people (Flores et al., 2016). Younger people (18-24) are more likely than older people to identify as transgender (Flores et al., 2016). The number of TGNC people seeking support from health professionals is growing (Reed et al., 2009). In the U.S., for example, the number of patients with a gender identity-related diagnostic code increased over three and a half fold between 2010 and 2014, from 3.87 to 14.22 per 100,000 patients (Canner et al., 2018).

Transgender health needs have become salient in the public consciousness in recent years. American public opinion is increasingly accepting of transgender people, due perhaps to greater visibility in popular culture and public life (Trotta, 2016; Steinmetz, 2014). Under the Obama Administration, there was considerable favorable policy momentum for the transgender community (Office of the Press Secretary, 2016). However, the Trump administration appears intent on reversing these gains by removing protections for TGNC people in health care (Pear, 2018). Despite increasing visibility and some political advancement, TGNC people continue to experience extensive health and social disparities.

In this comment article, we present an overview of the research evidence about the extent of TGNC health disparities. We then briefly review a sample of existing English language health communication campaigns relating to sex-specific cancersbreast, cervical, and testicular cancers-from a TGNC lens, to help identify gaps and opportunities for creating culturally competent health materials. Finally, we make a case for considering gender diversity in general, and TGNC people in particular, in health messaging.

\section{Health inequities and professional calls to action}

TGNC people face an elevated disease burden and significant health disparities (Mayer et al., 2008). For example, studies of transgender people on cross-sex hormone therapy have observed a higher prevalence of venous thrombosis, myocardial infarction, and cardiovascular disease among transgender women, polycythemia among transgender men, and increased insulin resistance and fasting glucose among both groups as compared to control populations (Gooren et al., 2008; Wierckx et al., 2013; Weinand and Safer, 2015). Transgender women have a very high HIV burden (Baral et al., 2013; James et al., 2016). The evidence of mental health disparities is mixed; the majority of studies suggest that TGNC people have poorer mental health outcomes than the population as a whole, while others observed no or marginal differences among subgroups such as sociallytransitioned transgender youth with parental support (James et al., 2016; Olson et al., 2016; Reisner et al., 2016; Valentine and Shipherd, 2018).

Small sample sizes and variations in aggregate gender minority groups within population-based studies currently limit the ability to report accurate incidence and prevalence of cancer among TGNC people (Quinn et al., 2015). Additionally, cancer registries do not document TGNC identity. While cross-sex hormone therapy is not thought to be associated with increased cancer prevalence and mortality, TGNC people engage in certain cancer risk behaviors, for example tobacco and alcohol use, at higher levels (Weinand and Safer, 2015; Quinn et al., 2015; James et al., 2016).

Several social determinants of health $(\mathrm{SDOH})$ contribute to TGNC health disparities such as social ostracism, discrimination, family rejection, poverty, homelessness, and violence (Lombardi et al., 2001; Bradford et al., 2013; James et al., 2016). A lack of access to affirming, appropriate health care is also concerning. Barriers to care include difficulty accessing culturally competent and adequately trained providers, insurance coverage exclusions for transition-related care, inaccessible and normatively gendered health care spaces, and a lack of trust in providers and health delivery systems due to discrimination and mistreatment (Poteat et al., 2013; Kosenko et al., 2013; James et al., 2016). Additionally, structural racism results in limited access to health care and poor health outcomes for TGNC people of color (James et al., 2016).

For many years, there have been calls within public health for increased research and data collection to address the specific health needs of lesbian, gay, bisexual, and transgender (LGBT) communities (Lombardi, 2001; Clark et al., 2001; Mayer et al., 2008; Daulaire, 2014). Yet, there remains a dearth of data available about these communities across all health fields (Boehmer, 2002; World Health Organization, 2013). The American Public Health Association adopted a policy statement 20 years ago addressing the need for research on sexual orientation and gender identity (American Public Health Association, 1998). Until recently, though, research addressing the health and health care needs of LGBT people predominantly focused on LGB communities, with the heaviest focus on gay men.

While research about TGNC people is growing, it is still relatively sparse. There have been calls in recent years for increased population-level transgender data collection to help monitor progress towards health objectives, including the Healthy People 2020 targets (U.S. Department of Health and Human Services, 2017). Research on intersectional, strengths-based approaches to promoting health have been suggested (Colpitts and Gahagan, 2016). More and better health research with TGNC communities is urgently needed.

There has been little examination of the role that health communication can play in improving TGNC health, but the existing literature highlights opportunities. For example, Drabble et al. (2003) call for more LGBT-focused research on health communication activities and involving LGBT communities in the development of population-specific health communication campaigns that account for their diversity. There are opportunities in the field of health communication, especially given the availability of innovative communication channels and audience segmentation methods, for targeted, culturally competent health messages to reach TGNC people as part of dedicated health campaigns.

\section{Health communications campaigns and audience segmentation}

Health communication campaigns that utilize marketing strategies can deliver specific messages to identified audiences to influence health behavior, directly, or indirectly. Campaigns can take many forms, and with the evolution of technology-mediated communication, the ways of reaching audiences are innumerable. Based primarily on health behavior theory, campaigns target a range of outcomes from increased knowledge to improved selfefficacy, changes in perceived threat or behavioral intention. Messages can appeal using logic or reasoning, but more often use pathos or ethos to connect specific behaviors with an existing or desired cultural or social norm. 
A primary strategy in health communication campaigns is audience segmentation, a process by which campaign developers analyze audience characteristics and narrow down to as specific and homogenous an audience as possible to tailor messaging based on their shared characteristics. The four typical categories considered in audience segmentation are geographic, demographic, psychographic, and behavioral (Kotler and Armstrong, 2001). The advent of technology that allows organizations to track specific information and behavior patterns of individuals (i.e., purchasing history, topics searched via the internet, types of material read, affinity groups) allows for very specific segmentation as well as targeted delivery of campaign messaging through channels primarily used by the identified segment. Thus, although the TGNC population is relatively small in number, there are specific social groups and communication outlets that are commonly known and used among the population that allow for costeffective targeted health communication to that community.

\section{Cancer campaign case studies}

As we examined cancer campaign case studies, we hypothesized that health communication campaigns on sex-specific conditions may exclude TGNC people if they have been designed for a gender-segmented cisgender ${ }^{2}$ audience. In other words, when advertisers use masculine or feminine stereotypes to appeal to cisgender people, these messages will almost certainly alienate their TGNC counterparts. To explore this, we searched the literature for messaging on sex-specific cancers and analyzed the ways in which gender has been used in campaigns. We present four brief case studies here. The first two case studies relate to messaging on breast and testicular cancer that target cisgender audiences. Then, we present the only campaigns known to use cancer messaging campaigns that engage TGNC people directlyone encouraging TGNC men to have a pap test and the other emphasizing breast health.

Case study 1: “The Pink Ribbon Campaign" (Susan G. Komen for the Cure, U.S.). Breast cancer is the most commonly diagnosed cancer among individuals assigned female at birth. One in eight of these individuals will be diagnosed with breast cancer in their lifetime (DeSantis et al., 2014). Over the age of 50, annual mammograms to detect breast cancer is indicated for trans masculine people, even after having a bilateral mastectomy (due to residual breast tissue), and for trans feminine people who have taken estrogen for 5-10 years (Gorton and Grubb, 2014; Center of Excellence for Transgender Health, 2016). Given the limited data and the lack of culturally tailored messaging, TGNC people and their doctors may not understand or even be aware of their risk.

Public health campaigns to raise awareness about breast cancer are often hyperfeminized, as exemplified by the ubiquitous Pink Ribbon Campaign. This campaign was launched by the Susan G. Komen Breast Cancer Foundation (now known as Susan G. Komen) in 1991. Focused on breast cancer, the foundation adopted pink, a color contemporarily associated with women and femininity, as its symbolic color at its inception in 1982. The majority of this and other breast cancer messaging remains branded in pink. Susan G. Komen's campaign messaging is deployed through a variety of channels such as advertisements, educational materials, local, and national fundraisers such as walks and galas, and branded merchandise. Corporate sponsors also utilize the pink branding in their philanthropic or social responsibility efforts.

TGNC people, including transgender men, are largely excluded by the campaign's heavily gendered, pink focus. Susan G. Komen has created educational materials for LGBT people, but missed an opportunity for specific education on risks to TGNC people due to the language used. Their LGBT fact sheet states that, "the two most common risk factors for breast cancer are being female (and) getting older," but this excludes TGNC people who do not identify as female but who still have a risk of breast cancer (Komen, 2016). This can be remedied by mentioning TGNC people explicitly and using more nuanced language in their targeted materials.

Beyond the campaigns themselves, communicating about breast health to TGNC populations requires further considerations. Oftentimes mammograms are performed in gendered spaces, such as women's centers or as part of women's programs, and communication about these services generally continues the feminine theme. However, women's spaces can be inaccessible for TGNC people. For trans masculine identified and/or presenting people, these spaces can be uncomfortable because they are discordant with their identities. There may be concerns about whether or not they will be welcomed and, above all, safe in gendered spaces. This may lead to discomfort or avoidance. Furthermore, as TGNC people medically transition, their doctors may not be aware of cancer screening recommendations for their bodies and they may be excluded from screening reminder programs that are segregated by sex.

Case study 2: "Know Your Nuts" (Testicular Cancer Awareness Foundation, U.S.). The efficacy of routine screening for testicular cancer has not been established. Screening has not been shown to reduce mortality and the disease is usually treatable at any stage (U.S. Preventative Services Task Force, 2011; National Cancer Institute, 2012; Gorton and Grubb, 2014). Routine screening for asymptomatic transgender women is not recommended at this time (Center of Excellence for Transgender Health, 2016). However, testicular cancer is often found by doctors as part of a routine physical exam or by patients themselves, and some doctors recommend monthly self-exams after puberty (American Cancer Society, 2016).

TGNC people who have not had an orchiectomy and experience symptoms may need to have a testicular exam. This exam should be conducted with care to ensure patient psychological comfort (Potter et al., 2015; Center of Excellence for Transgender Health, 2016). Patients may also benefit from general information about testicular cancer. All information given to TGNC patients should use gender-neutral language where possible and, ideally, be tailored to a TGNC audience. Culturally sensitive guidance is needed, but to our knowledge it is not currently available.

Imagery in the Testicular Cancer Awareness Foundation's "Know Your Nuts" campaign to encourage screening uses gendered messaging to target general male audiences. Campaign ads feature images of "balls" and language of "nuts"- both common slang terms for testicles. About half of testicular cancer cases are diagnosed between the ages of 20-34, and the tone of the messaging reflects the campaign's young target audience. A similar campaign was run by the Testicular Cancer Foundation during June 2016 for Men's Health Month, using the hashtag "\#KnowYourBalls" accompanied by basketball imagery. These campaigns are deployed primarily through placement in magazines such as Men's Health and through social media.

The campaigns clearly target young cisgender men. Transgender women and GNC people who have testicles may or may not respond favorably to sports imagery, but combined with masculinized language and the location and method of deployment, these campaigns are unlikely lead to health behavior change in the TGNC population. Moreover, the association of testicular cancer with masculinized imagery, in light of the 
complicated relationship many TGNC people have with their gendered bodies, may dissuade them from pursuing screening services for testicular health.

Case study 3: "Paps Matter for Trans Men" (Rainbow Health Ontario/Sherbourne Health Center/Community One Foundation, Toronto, Canada). TGNC people who have a cervix should have regular pap tests following the same guidance as cisgender women and, as with testicular and breast exams, providers should take extra care to use affirming language and empower the patient (Gorton and Grubb, 2014; Potter et al., 2015). The "Paps Matter for Trans Men" campaign, launched in 2010 by Rainbow Health Ontario, uses images of young to middle-age trans masculine people of different racial and ethnic groups. They are accompanied by phrases such as "Guys Get Paps Too" and "Paps are Worth It." In each picture, one of the models has a tattoo that says, "Check It Out Guys." The ads were developed by and for transgender men and their health care providers and were primarily deployed through transgender health organizations and LGBT social networks.

This campaign received a positive response from the transgender community. The Check It Out Guys Website (www.checkitoutguys.ca) was voted Best Action Campaign in the 2010 TransGuys.com Community Awards. The campaign narrowly targeted a young trans masculine audience. Future steps could include building a campaign that addresses other parts of the community such as older transgender men and TGNC people who were assigned female at birth and have diverse gender presentations. To the authors' knowledge, this is currently the only published public health campaign about cancer that targets trans masculine people directly.

Case study 4: "Love Your Breasts. Or Lose Your Breasts." (Stirred Creative/HCG/Pink Hope Patient Support Group, India). The "Love Your Breasts. Or Lose Your Breasts." poster and billboard campaign was launched in 2014 by HealthCare Global Oncology (HCG), a cancer care network in India (Stirred Creative, 2014). The campaign, created in partnership with the transgender community in Bangalore, purports that transgender women protect and value their femininity and therefore get their breasts checked regularly to avoid having to undergo a mastectomy. Visual messages were displayed on billboards, and were accompanied by a film and the launch of a breast cancer app. According to the campaign's creator, its purpose was to "shake the average [cisgender] woman out of her apathy towards regular breast checks" by highlighting the attention that transgender women, a "fairly marginalized community in India," give to their breast health. He reported that the medical community was initially skeptical but the initiative quickly gained their support and the campaign won several accolades in India (E Anthony 2015, personal communication, 23 October).

Data on transgender women and TGNC people assigned male at birth and breast cancer is limited but, as discussed previously, screening mammography is recommended for some thought to be at higher risk. One critique of the "Love Your Breasts. Or Lose Your Breasts." campaign, purely from a TGNC health point of view, is that it uses transgender women to make a point about cisgender women's health rather than addressing TGNC health directly. However, a doctor from HCG noted in the press that transgender women are also at risk and should conduct breast self-examination, therefore, awareness may have increased as a byproduct of the campaign (Kellaway, 2015). Just as the "Paps Matter" campaign was the only example of a cancer public health campaign targeting transgender men, so too this was the only one featuring transgender women.

\section{The importance of considering TGNC people in health communication campaigns}

For nearly two decades, the American Public Health Association has acknowledged that more research and better access to adequate health care is needed to reduce health disparities faced by the transgender community; however, our review found that very few public health messages outside of HIV and anti-violence campaigns directly target TGNC people. Indeed, mainstream campaigns may further marginalize this population due to their gendered language or imagery. The relative absence of existing inclusive campaigns points to an opportunity for health communication professionals to learn about and engage with TGNC communities in new and exciting ways.

Public health campaigns providing messaging about sexspecific cancers (e.g., testicular and breast cancer) and sexual health often rely upon gendered and stereotypical masculine and feminine messaging to reach the general public. These frameworks fail to recognize the complexities of the lived experiences of TGNC people and their bodies. This results in a public health landscape wherein TGNC people may not be adequately informed of their risk and underutilize health care services, leading to both late diagnosis and treatment (Lerner and Robles, 2017).

Opportunities exist for public health professionals to explore and respond to the health needs of TGNC people across their diverse communities. Local, regional, and national/federal health departments should acknowledge the existence of TGNC people in their communities, commit to learning about the distinct health care needs of these populations, and dedicate resources to reduce barriers to health and health care. This includes not only addressing health concerns where TGNC people are at a higher risk such as HIV or gender-based violence, but also general health concerns such as cancer risk, smoking, nutrition, and physical activity. Thus, health departments should prioritize the development of health promotion materials to reach TGNC people across the communities they serve.

Any public health campaign attempting to address the health needs of TGNC people should be developed alongside the community to ensure cultural relevance ${ }^{3}$. Local health departments should establish community advisory boards that have meaningful influence over their department's strategic plans and health communication campaigns. These efforts can serve to best advance health equity where authentic community engagement is established. Advisory boards with diverse representation of community members can help to establish plans and campaigns that more accurately reflect and meet the needs of residents.

As with other marginalized communities, TGNC people rely upon health professionals to help identify their general and unique risks. Health professionals must acknowledge that cancer screenings for TGNC people can be a considerably more difficult and uniquely vulnerable experience, depending on a person's gender history, their body, and the contexts in which these encounters take place.

\section{Conclusion}

To meet the needs of the growing TGNC community, the public health profession must either make existing health communication campaigns more inclusive of gender diversity or become more expansive in its outreach to, and engagement of, TGNC people by creating targeted campaigns. Campaign developers should consider the diversity of the communities in which the campaigns are situated as well as the diversity within TGNC communities reflecting characteristics such as race and ethnicity, ability, age, and gender presentation. 
Community-based participatory research may aid in the development of culturally competent campaigns. TGNC people should be consulted from the outset and should drive the direction of health message development to ensure accuracy and relevance. Health promotion materials using communityinformed language and images will increase the likelihood that the messaging will meet the needs of this audience.

Finally, thinking broadly, health departments at all levels should learn more about the specific health issues affecting TGNC people in their community. To aid this effort, health departments should research best practices for survey question formation and communication and ensure the collection of population-level demographics data includes gender identity. For analysis, these data should be disaggregated by race and other demographic variables to capture the distinct experiences and risk factors associated with intersecting inequities. Departments should also require training for their employees regarding TGNC identities, health inequities, and health and community needs in a social determinants of health and health equity framework. Only by being considered at all levels and included in health communication campaigns can TGNC people attain the best possible health.

Received: 8 May 2018 Accepted: 24 July 2018

Published online: 14 August 2018

\section{Notes}

1 We have been intentional about our use of transgender vs. transgender and gender nonconforming (TGNC) in this piece. The literature varies in its definition of who counts as transgender and whether the definition includes people who identify as nonbinary, gender diverse, genderqueer, et cetera. Research on gender nonconforming people's health needs specifically is scarce and, more often than not, gender nonconforming people are not explicitly mentioned. However, we believe they are important to include in any analysis of gendered public health messaging.

2 Cisgender refers to people whose gender identity matches with the sex they were assigned at birth. The Latin root word cis means "on this side of."

3 Other work concerning TGNC people and cancer prevention is the "HPV \& Guys Like Me" study (Xian Brooks MPH), which aimed to conduct and advance reproductive health and reproductive cancer prevention research, as well as provide education for trans masculine and gender nonconforming people. The investigator took a collaborative, community-based approach. See: http://www.nchrc.org/assets/TransHealth/hpv-and-guys-like-me-zine-UPDATED.pdf. Pioneering work has also been done on the psychosocial impact of cancer on LGBTQ people (Taylor and Bryson, 2016). See: http://www.lgbtcancer.ca/ .

\section{References}

American Cancer Society (2016) Do I have testicular cancer? [online] Cancer.org. Available at: http://www.cancer.org/cancer/testicularcancer/ moreinformation/doihavetesticularcancer/do-i-have-testicular-cancer-selfexam. [accessed 28 Apr 2018]

American Public Health Association (1998) The need for public health research on gender identity and sexual orientation. [online] APHA.org. Available at: http://www.apha.org/policies-and-advocacy/public-health-policy-statements/ policy-database/2014/07/29/14/32/the-need-for-public-health-research-ongender-identity-and-sexual-orientation. [accessed 28 Apr 2018]

Baral SD, Poteat T, Strömdahl S, Wirtz AL, Guadamuz TE, Beyrer C (2013) Worldwide burden of HIV in transgender women: a systematic review and meta-analysis. Lancet Infect Dis 13(3):214-22. https://doi.org/10.1016/S14733099(12)70315-8

Boehmer U (2002) Twenty years of public health research: inclusion of lesbian, gay, bisexual, and transgender populations. Am J Public Health 92(7):1125-1130. https://doi.org/10.2105/AJPH.92.7.1125

Bradford J, Reisner SL, Honnold JA, Xavier J (2013) Experiences of transgenderrelated discrimination and implications for health: results from the Virginia transgender health initiative study. Am J Public Health 103(10):1820-1829. https://doi.org/10.2105/AJPH.2012.300796

Canner JK, Harfouch O, Kodadek LM, Palaez D, Coon D, Offodile AC, Haider AH, Lau BD (2018) Temporal trends in gender-affirming surgery among transgender patients in the United States. JAMA Surg 153(7):609-616. https://doi.org/10.1001/jamasurg.2017.6231

Center of Excellence for Transgender Health (2016) Guidelines for the primary and gender-affirming care of transgender and gender nonbinary people, 2nd edn [online]. Transhealth.ucsf.edu. Available at: http://transhealth.ucsf.edu/pdf/ Transgender-PGACG-6-17-16.pdf. [accessed 18 Jul 2018]

Clark ME, Landers S, Linde R, Sperber J (2001) The GLBT health access project: a state-funded effort to improve access to care. Am J Public Health 91 (6):895-896. https://doi.org/10.2105/AJPH.91.6.895

Daulaire N (2014) The importance of LGBT health on a global scale. LGBT Health 1(1):8-9. https://doi.org/10.1089/lgbt.2013.0008

DeSantis C, Ma J, Bryan L, Jemal A (2014) Breast Cancer Statistics, 2013. CACancer J Clin 64(1):52-62. https://doi.org/10.3322/caac. 21203

Drabble L, Keatley J, Marcelle G (2003) Progress and opportunities in lesbian, gay, bisexual, and transgender health communications. Clin Res Regul Aff 20 (2):205-227. https://doi.org/10.1081/CRP-120021081

Flores AR, Herman JL, Gates GJ and Brown TNT (2016) How many adults identify as transgender in the United States? [online] UCLA.edu. Available at: https:// williamsinstitute.law.ucla.edu/wp-content/uploads/How-Many-AdultsIdentify-as-Transgender-in-the-United-States.pdf. [accessed 28 April 2018]

Gooren LJ, Giltay EJ, Bunck MC (2008) Long-term treatment of transsexuals with cross-sex hormones: extensive personal experience. J Clin Endocrinol Metab 93(1):19-25. https://doi.org/10.1210/jc.2007-1809

Gorton N, Grubb HM (2014) General, sexual, and reproductive health. In: Erickson-Schroth L (ed). Trans Bodies, Trans Selves: A Resource For The Transgender Community. Oxford University Press, New York, NY, pp. 215-240

James SE, Herman JL, Rankin S, Keisling M, Mottet L, Anafi M (2016) The report of the 2015 U.S. Transgender Survey. [online] Transequality.org. Available at: http://www.transequality.org/sites/default/files/docs/USTS-Full-ReportFINAL.PDF. [accessed 28 Apr 2018]

Kellaway M (2015) PHOTOS: Indian trans women join the fight against breast cancer, The Advocate, 9 Jan, Available at: https://www.advocate.com/health/ 2015/01/09/photos-indian-trans-women-join-fight-against-breast-cancer [accessed 19 Jul 2018]

Kosenko K, Rintamaki L, Raney S, Maness K (2013) Transgender patient perceptions of stigma in health care contexts. Med Care 51(9):819-22. https:// doi.org/10.1097/MLR.0b013e31829fa90d

Kotler P, Armstrong G (2001) Principles of Marketing, 9th edn. Pearson Prentice Hall, Saddle River

Lerner JE, Robles G (2017) Perceived barriers and facilitators to health care utilization in the United States for transgender people: A review of recent literature. J Health Care Poor Under 28(1):127-152. https://doi.org/10.1353/ hpu.2017.0014

Lombardi E (2001) Enhancing transgender health care. Am J Public Health 91 (6):869-872

Lombardi EL, Wilchins RA, Priesing D, Malouf D (2001) Gender violence: transgender experiences with violence and discrimination. J Homosex 42 (1):89-101. https://doi.org/10.1300/J082v42n01_05

National Cancer Institute (2012) Testicular cancer screening $\left(\mathrm{PDQ}^{\circledast}\right)$. [online] Cancer.gov. Available at: http://www.cancer.gov/types/testicular/patient/ testicular-screening-pdq\#section/_19. [accessed 28 Apr 2018]

Mayer KH, Bradford JB, Makadon HJ, Stall R, Goldhammer H, Landers S (2008) Sexual and gender minority health: what we know and what needs to be done. Am J Public Health 98(6):989-995. https://doi.org/10.2105/ AJPH.2007.127811

Olson KR, Durwood L, DeMeules M, McLaughlin KA (2016) Mental health of transgender children who are supported in their identities. Pediatrics 137(3): e20153223. https://doi.org/10.1542/peds.2015-3223

Office of the Press Secretary (2016) Fact Sheet: Obama Administration's Record and the LGBT Community. [online] Archives.gov. Available at: https:// obamawhitehouse.archives.gov/the-press-office/2016/06/09/fact-sheetobama-administrations-record-and-lgbt-community. [accessed 28 Apr 2018]

Pear R (2018) Trump plan would cut back health care protections for transgender people. [online] NYTimes.com. Published 21 Apr. Available at: https://www. nytimes.com/2018/04/21/us/politics/trump-transgender-health-care.html [accessed 28 Apr 2018]

Poteat T, German D, Kerrigan D (2013) Managing uncertainty: a grounded theory of stigma in transgender health care encounters. Soc Sci Med 84:22-9. https:// doi.org/10.1016/j.socscimed.2013.02.019

Potter J, Peitzmeier SM, Bernstein I, Reisner SL, Alizaga NM, Agenor M, Pardee DJ (2015) Cervical cancer screening for patients of the female-to-male spectrum: a narrative review and guide for clinicians. J Gen Intern Med 30(12):1857-64. https://doi.org/10.1007/s11606-015-3462-8

Quinn GP, Sanchez JA, Sutton SK, Vadaparampil ST, Nguyen GT, Green BL Kanetsky PA, Schabath MB (2015) Cancer and lesbian, gay, bisexual, transgender/transsexual, and queer/questioning (LGBTQ) populations. CA Cancer J Clin 65(5):384-400. https://doi.org/10.3322/caac.21288 
Reed B, Rhodes S, Schofield P, Wylie K (2009) Gender variance in the UK: Prevalence, incidence, growth and geographic distribution. [online] Gires.org.uk. Available at: http://www.gires.org.uk/assets/Medpro-Assets/ GenderVarianceUK-report.pdf. [accessed 28 Apr 2018]

Reisner SL, Biello KB, White Hughto JM, Kuhns L, Mayer KH, Garafalo R, Mimiaga MJ (2016) Psychiatric diagnoses and comorbidities in a diverse, multicity cohort of young transgender women: Baseline findings from project lifeskills. JAMA Pediatr 170(5):481-486. https://doi.org/10.1001/ jamapediatrics.2016.0067

Stirred Creative (2014) HCG: About the campaign. [online] Stirredcreative.com. Available at: https://www.stirredcreative.com/hcg-case-study. [accessed 28 Apr 2018]

Steinmetz K (2014) The transgender tipping point. [online] Time.com. Published 29 May. Available at: http://time.com/135480/transgender-tipping-point/. [accessed 28 Apr 2018]

Susan G. Komen (2016) Facts for life: lesbian, gay, bisexual and transgender people. [online] Komen.org. Available at: https://ww5.komen.org/uploadedFiles/ _Komen/Content/About_Breast_Cancer/Tools_and_Resources/

Fact_Sheets_and_Breast_Self_Awareness_Cards/Lesbian,\%20Gay,\% 20Bisexual\%20and\%20Transgender\%20People.pdf [accessed 28 Apr 2018]

Taylor ET, Bryson MK (2016) Cancer's margins: Trans* and gender nonconforming people's access to knowledge, experiences of cancer health, and decision-making. LGBT Health 3(1):79-89. https://doi.org/10.1089/ lgbt.2015.0096

Trotta D (2016) Exclusive: Women, young more open on transgender issue in U.S. - Reuters/Ipsos poll. [online] Reuters.com. Published 21 Apr. Available at: http://www.reuters.com/article/us-usa-lgbt-poll-idUSKCNOXI11M. [accessed 28 Apr 2018]

U.S. Department of Health \& Human Services (2017) Lesbian, gay, bisexual, and transgender health. [online] Healthypeople.gov. Available at: https://www. healthypeople.gov/2020/topics-objectives/topic/lesbian-gay-bisexual-andtransgender-health/objectives. [accessed 28 Apr 2018]

U.S. Preventive Services Task Force (2011) Screening for testicular cancer: U.S. Preventive Services Task Force reaffirmation recommendation statement. Ann Intern Med 154:483-486. https://doi.org/10.7326/0003-4819-154-7201104050-00006

Valentine SE, Shipherd JC (2018) A systematic review of social stress and mental health among transgender and gender non-conforming people in the United States. Clin Psychol Rev. pii: S0272-7358(17)30420-8. https://doi.org/10.1016/ j.cpr.2018.03.003. [epub ahead of print]

Wierckx K, Elaut E, Declercq EV, Heylens G, De Cuypere G, Taes Y, Kaufman JM, T'Sjoen G (2013) Prevalence of cardiovascular disease and cancer during cross-sex hormone therapy in a large cohort of trans persons: a case-control study. Eur J Endocrinol 169(4):471-8. https://doi.org/10.1530/EJE-13-0493

World Health Organization (WHO) (2013) Improving the health and well-being of lesbian, gay, bisexual and transgender persons: Report by the secretariat. [online] GHwatch.org. Available at: http://www.ghwatch.org/sites/www. ghwatch.org/files/B133-6_LGBT.pdf. [accessed 28 Apr 2018]

\section{Acknowledgements}

We would like to acknowledge the contributions of our colleagues at the Commonwealth Institute of Kentucky. In particular, we would like to thank Baraka Muvuka and Carmen Mitchell for their assistance with this article, and Nida Ali and Trinidad Jackson for their contributions to the poster that preceded this manuscript. We would also like to thank Stephanie Boone for her helpful feedback.

\section{Additional information}

Competing interests: The authors declare no competing interests.

Reprints and permission information is available online at http://www.nature.com/ reprints

Publisher's note: Springer Nature remains neutral with regard to jurisdictional claims in published maps and institutional affiliations.

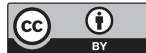

Open Access This article is licensed under a Creative Commons Attribution 4.0 International License, which permits use, sharing, adaptation, distribution and reproduction in any medium or format, as long as you give appropriate credit to the original author(s) and the source, provide a link to the Creative Commons license, and indicate if changes were made. The images or other third party material in this article are included in the article's Creative Commons license, unless indicated otherwise in a credit line to the material. If material is not included in the article's Creative Commons license and your intended use is not permitted by statutory regulation or exceeds the permitted use, you will need to obtain permission directly from the copyright holder. To view a copy of this license, visit http://creativecommons.org/ licenses/by/4.0/.

(C) The Author(s) 2018 